\title{
OXIDATION KINETICS AND MECHANISTIC STUDIES OF THIOACIDS BY IMIDAZOLIUM DICHROMATE
}

\author{
Ruchi Kalla, Devendra Kumar, Anurag Choudhary ${ }^{\varpi}$ and Vinita Sharma \\ Chemical Kinetics Laboratories, Department of Chemistry, J.N.V. University, \\ Jodhpur-432 005 (Rajasthan) India \\ ${ }^{\square}$ Corresponding Author: drpkvs27@yahoo.com
}

\begin{abstract}
We have studied three thioacids(TA) namely; thio-glycollic(TGA), thio-lactic(TLA) and thio-malic(TMA) acid by imidazoliumdichromate (IDC) in dimethyl-sulphoxide (DMSO). Oxidation of these thio-acids leads to the formation of di-sulphide di-mmers. The reaction is having a unit order with the IDC. A fractional-order reaction was found w.r.t. TA. Test for free radical was not observed in this reaction. The influence of acidity was observed, which indicated the increase in the rate by the addition of acid. The study of oxidation of TLA was done in various nonaqueous media, and the influence of solvent media was examined by using some multi-parametric equation models. We postulate a mechanism in which a thio-eater is formed, which is decomposing in the rate-determining slow step. Keywords: Dichromate, Oxidation Kinetics, Thioacid, Solvent Effects
\end{abstract}

RASĀYAN J. Chem., Vol. 14, No.4, 2021

\section{INTRODUCTION}

The majority of chromium compounds that have already been used in the synthesis of organic compounds are highly reactive and can't be selective as mild reagents. It has been noticed that these reagents are not soluble in the majority of solvent media. To prevail over the restrictions, various derivatives of $\mathrm{Cr}(\mathrm{VI})$ derivatives were introduced in synthetic organic chemistry as a soft and careful oxidizing reagent in nonaqueous solvents. ${ }^{1-4}$ A dichromate of imidazolium (IDC) is also one of the compounds available for this purpose. ${ }^{5}$ A literature survey indicated the presence of only some of the reports on oxidation aspects. ${ }^{6-9}$ In the present manuscript we are presenting the oxidation kinetics TGA, TLA and TMA by IDC in dimethylsulphoxide using as a solvent medium. A suitable mechanism has also been proposed here.

\section{EXPERIMENTAL}

All the thio-acids used in this investigation are available in the market (Sigma Chemicals and Fluka) and are ready to use chemicals. The thioacids (Fluka) and dithiodiglycollic acid (, USA) were commercial products and were used as such. The solutions of di-thiodimalic and di-thiodilactic acid are prepared by reported method. ${ }^{10}$ This method provides almost $99 \%$ pure acids. Solutions of these acids are prepared at the time of experiments in DMSO and the standardization was done iodimetrically. ${ }^{10-11}$ IDC is synthesized by the method ${ }^{5}$ available in the literature and iodometric method was used to check its purity. Usual methods ${ }^{12}$ are used to purify the other chemicals. Toluene para sulphonic acid (PTS) is used for acid effect.

\section{Stoichiometry}

Polarigraphic ${ }^{13-14}$ methods are used for product analysis. It is observed that the cathode wave of the product matches with a known sample of di-sulphide dimmer. The reaction revealed a 1:2 stoichiometric determination means two molecules of thio-acid are going to oxidize the one molecule of IDC, which $\mathrm{s}$ going to be reduced. A reaction mixture having an extra amount of IDC is allowed to complete the reaction and the residual amount of IDC was found out by the iodometric method. These results also showed 1: 2 stoichiometry. After completion of the reaction, the oxidation number of chromium in the reduced reactions mixtures is found out by iodometric titrations. It is $3.90 \pm 0.10$.

$$
2 \mathrm{R}-\mathrm{S}-\mathrm{H}+\mathrm{Cr}_{2} \mathrm{O}_{7}^{-2}+12 \mathrm{H}^{+} \longrightarrow \mathrm{R}-\mathrm{S}-\mathrm{S}-\mathrm{R}+7 \mathrm{H}_{2} \mathrm{O}+2 \mathrm{Cr}^{+3}
$$


RASĀYAN $J$. Chem.

Vol. 14 | No. 4 |2436-2441| October- December | 2021

\section{Kinetics Parameters}

Our reaction conditions are of pseudo-first-order in which we keep a ratio of 1:15 of reductant over oxidant. Medium is dimethysulphoxide in all the experiments except those of solvent effect where different solvents are used in the study. The reactions are performed in dark flasks to avoid the photochemical reactions and are done up to 2 half-lives by monitoring the decrease in the [IDC] at 354 $\mathrm{nm}$ on a spectrophotometric device of AIMIL. The IDC showed a $\lambda_{\max }$ of $361 \mathrm{~nm}$. Further, no other species of chromium is having the absorbing on this particular wavelength. The pseudo-first-order rate constants, $\mathrm{k}_{\mathrm{obs}}$, are calculated from the linear least squares graphs of log [IDC] against time. Reproducibility of the kinetic data within $\pm 4 \%$. The second-order rate constants are calculated by $\mathrm{k}_{\mathrm{obs}} /[$ reductant]. All the reactions are performed in the absence of acids, except those which were specifically done to see the effect of acid.

\section{RESULTS AND DISCUSSION}

\section{Rate Laws}

The reactions so done are of unit order with oxidant, and first-order rate constants are not depending on the starting concentrations of the IDC. As we increase the concentration of TA, the rate of reaction also increases but is not linearly related, as shown by the data in table one and figure one also, which is a specific kinetic graph. A plot of $1 / \mathrm{k}_{\mathrm{obs}}$ versus $1 /[\mathrm{TA}]$ is a linear graph $(\mathrm{r}>0.995)$ that has an intercept on the rate-ordinate (Fig.-2). Therefore, a fractional-order kinetic picture is obtained. This may lead to the following mechanism and the rate laws as equations 2 and 3 and 4.

$$
\begin{aligned}
& \mathrm{K} \\
& \text { Thioacid + IDC } \leftrightarrows \quad[\text { complex }] \\
& k_{2} \\
& \text { [Complex] } \rightarrow \text { Product } \\
& \text { Rate }=k_{2} \mathrm{~K}[\mathrm{TA}][\mathrm{IDC}] /(1+\mathrm{K}[\mathrm{TA}])
\end{aligned}
$$

Table-1: Rate Constant of Oxidation of TA by IDC at $298 \mathrm{~K}$ Temperature

\begin{tabular}{c|c|c|c|c}
\hline $10^{3}[$ IDC $]$ & {$[$ Thioacid] } & \multicolumn{3}{|c}{$10^{4} k_{\text {obs }}\left(\mathrm{s}^{-1}\right)$} \\
\cline { 3 - 5 }$\left(\mathrm{mol} \mathrm{dm}^{-3}\right)$ & $\left(\mathrm{mol} \mathrm{dm}^{-3}\right)$ & TGA & TLA & TMA \\
\hline 1.0 & 0.10 & 4.85 & 20.2 & 10.5 \\
\hline 1.0 & 0.20 & 7.26 & 30.3 & 15.7 \\
\hline 1.0 & 0.40 & 9.65 & 40.6 & 20.7 \\
\hline 1.0 & 0.60 & 10.8 & 45.7 & 23.2 \\
\hline 1.0 & 0.80 & 11.5 & 48.8 & 24.7 \\
\hline 1.0 & 1.00 & 12.0 & 50.9 & 25.7 \\
\hline 1.0 & 1.50 & 12.7 & 53.9 & 27.1 \\
\hline 1.0 & 3.00 & 13.5 & 57.3 & 28.8 \\
\hline 2.0 & 0.40 & 9.18 & 39.6 & 19.8 \\
\hline 4.0 & 0.40 & 9.72 & 41.4 & 21.6 \\
\hline 6.0 & 0.40 & 9.36 & 40.5 & 19.0 \\
\hline 8.0 & 0.40 & 9.54 & 42.3 & 20.5 \\
\hline 1.0 & 0.20 & $7.47^{*}$ & $31.5^{*}$ & $16.2^{*}$ \\
\hline${ }^{\text {a }}$ contained $0.001 \mathrm{M}$ acrylonitrile
\end{tabular}

Table-2: Effect of Hydrogen ion Concentration on the Rate of TA by I.D.C.

\begin{tabular}{c|c|c|c|c|c|c}
\hline \multicolumn{2}{c}{ [T.D.C. $]=0.001 \mathrm{~mol} \mathrm{dm}^{-3}$} & \multicolumn{2}{c}{ Thioacids] $=1.0 \mathrm{~mol} \mathrm{dm}^{-3}$} & \multicolumn{2}{c}{ Temp. $=298 \mathrm{~K}$} \\
\hline$\left[\mathrm{H}^{+}\right] / \mathrm{mol} \mathrm{dm}^{-3}$ & 0.10 & 0.20 & 0.40 & 0.60 & 0.80 & 1.00 \\
\hline TGA & 5.67 & 7.02 & 8.10 & 9.45 & 12.3 & 13.1 \\
\hline TLA & 23.4 & 27.0 & 36.0 & 37.8 & 50.4 & 56.7 \\
\hline TMA & 12.6 & 14.4 & 18.0 & 21.6 & 26.1 & 29.7 \\
\hline
\end{tabular}


RASĀYAN J. Chem.

Vol. 14 | No. 4 |2436-2441| October- December | 2021

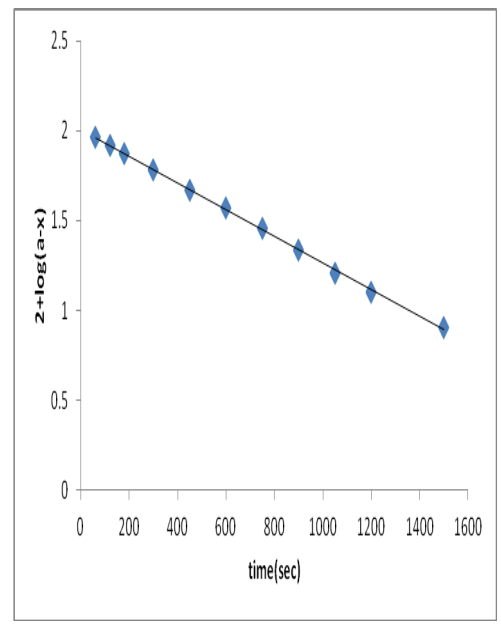

Fig.-1: A Graph showing Oxidation of TLA by IDC

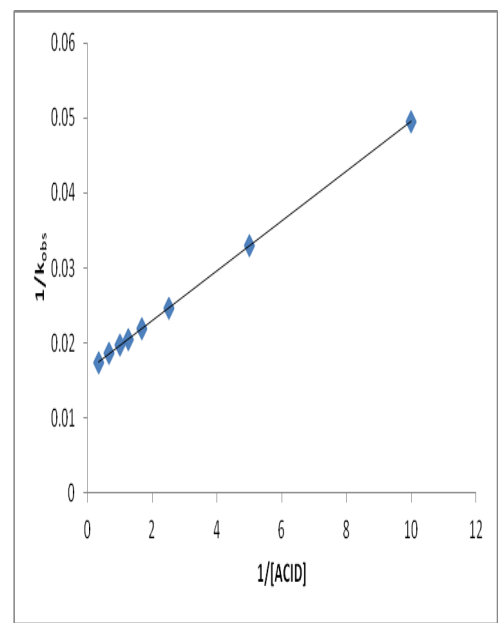

Fig.-2: Oxidation of TLA by IDC: A Double Reciprocal Plot

Studies at four different temperatures are done to see how reaction rates depend upon concentrations of TA. And the values of formation and decomposition constants are calculated from the graph given in Fig.-2. Activation parameters of decomposition of the complexes and thermodynamic parameter of formation of complexes are recorded in table numbers 2 and 3.

Table-3: Rate Constants and Activation Parameters of Decomposition of IDC-TA Complex

\begin{tabular}{|c|c|c|c|c|c|c|c|}
\hline \multirow[t]{2}{*}{ Thioacid } & \multicolumn{4}{|c|}{$10^{3} k_{2} /\left(\mathrm{dm}^{-3} \mathrm{~mol}^{-1} \mathrm{~s}^{-1}\right)$} & \multirow{2}{*}{$\begin{array}{c}\Delta H^{*} \\
\left(\mathrm{~kJ} \mathrm{~mol}^{-1}\right)\end{array}$} & \multirow{2}{*}{$\begin{array}{c}-\Delta S^{*} \\
\left(\mathrm{~J} \mathrm{~mol}^{-1} \mathrm{~K}^{-1}\right)\end{array}$} & \multirow{2}{*}{$\begin{array}{c}\Delta G^{*} \\
\left(\mathrm{~kJ} \mathrm{~mol}^{-1}\right)\end{array}$} \\
\hline & $288 \mathrm{~K}$ & $298 \mathrm{~K}$ & $308 \mathrm{~K}$ & $318 \mathrm{~K}$ & & & \\
\hline TGA & 7.29 & 14.4 & 26.1 & 50.4 & $46.1 \pm 0.7$ & $126 \pm 3$ & $83.5 \pm 0.6$ \\
\hline TLA & 36.0 & 61.2 & 108 & 171 & $37.4 \pm 0.5$ & $143 \pm 2$ & $79.9 \pm 0.4$ \\
\hline TMA & 17.1 & 20.6 & 54.9 & 95.4 & $41.2 \pm 0.4$ & $136 \pm 1$ & $81.6 \pm 0.3$ \\
\hline
\end{tabular}

Free Radical Test

To find out the presence of free radicals in the present reaction of TA and IDC, reactions are performed inert ambiance by nitrogen. It is observed that no precipitation is thereby adding vinyl chloride, indicating the absence of free radical mechanism is not operating in the present reaction. Data are already shown in table one.

\section{Hydrogen Ion Effect}

To see the effect of acidity, reaction are done under the influence of an acid. Reaction shows the effect of acidity with a relation $k_{\mathrm{obs}}=a+b\left[\mathrm{H}^{+}\right]$. Data for $a$ and $b$, of TGA, is $4.96 \pm 0.38 \times 10^{-3} \mathrm{~s}^{-1}$ and $8.34 \pm 1.63$ $\times 10^{-3} \mathrm{~mol}^{-1} \mathrm{dm}^{3} \mathrm{~s}^{-1}(r=0.9889)$.

\section{Influence of Solvent Medium}

Nineteen different solvents are used to see the effect on the rate of oxidation of T.L.A. by I.D.C. The data for $\mathrm{K}$ and $\mathrm{k}_{2}$ for T.L.A. - I.D.C. complexes at $298 \mathrm{~K}$ temperature is presented in table four. Not much variation was observed in the values of $K$ rather than the values $k_{2}$ have considerable variation.

Table-4: Formation Constants for the Decomposition of IDC-thioacids Complexes and Their Thermodynamic Parameters

\begin{tabular}{c|c|c|c|c|c|c|c}
\hline \multirow{2}{*}{ Thioacid } & \multicolumn{4}{|c|}{$\mathrm{K} /\left(\mathrm{dm}^{-3} \mathrm{~mol}^{-1} \mathrm{~s}^{-1}\right)$} & \multirow{2}{*}{$\begin{array}{c}-\Delta H^{*} \\
\left(\mathrm{~kJ} \mathrm{~mol}^{-1}\right)\end{array}$} & $\begin{array}{c}-\Delta S^{*} \\
\left(\mathrm{~J} \mathrm{~mol}^{-1} \mathrm{~K}^{-1}\right)\end{array}$ & $\begin{array}{c}-\Delta G^{*} \\
\left(\mathrm{~kJ} \mathrm{~mol}^{-1}\right)\end{array}$ \\
\cline { 2 - 5 } & $288 \mathrm{~K}$ & $298 \mathrm{~K}$ & $308 \mathrm{~K}$ & $318 \mathrm{~K}$ & $16.1 \pm 0.8$ & $33 \pm 3$ & $6.46 \pm 0.6$ \\
\hline TGA & 5.85 & 5.08 & 4.25 & 3.42 & $16.0 \pm 0.6$ & $33 \pm 2$ & $6.41 \pm 0.5$ \\
\hline TLA & 5.76 & 4.92 & 4.14 & 3.36 & $15.6 \pm 0.7$ & $31 \pm 2$ & $6.55 \pm 0.5$ \\
\hline TMA & 6.03 & 5.25 & 4.41 & 3.60 & \\
\hline
\end{tabular}


RASĀYAN $J$. Chem.

Vol. 14 | No. 4 |2436-2441| October- December | 2021

Table-5: Effect of Solvents on the Oxidation of TLA-IDC Complex at $298 \mathrm{~K}$

\begin{tabular}{c|c|c|c|c|c}
\hline Solvents & $\begin{array}{c}\mathrm{K} \\
\left(\mathrm{dm}^{-3} \mathrm{~mol}^{-1}\right)\end{array}$ & $\begin{array}{c}10^{4} \mathrm{k}_{\text {obs }} \\
\left(\mathrm{s}^{-1}\right)\end{array}$ & Solvents & $\begin{array}{c}\mathrm{K} \\
\left(\mathrm{dm}^{-3} \mathrm{~mol}^{-1}\right)\end{array}$ & $\begin{array}{c}10^{4} \mathrm{k}_{\text {obs }} \\
\left(\mathrm{s}^{-1}\right)\end{array}$ \\
\hline $\mathrm{CHCl}_{3}$ & 5.88 & 49.0 & $\mathrm{C}_{6} \mathrm{H}_{5} \mathrm{CH}_{3}$ & 4.99 & 15.5 \\
\hline $1,2-\mathrm{C}_{2} \mathrm{H}_{4} \mathrm{Cl}_{2}$ & 6.03 & 57.5 & $\mathrm{C}_{6} \mathrm{H}_{5} \mathrm{COCH}_{3}$ & 5.33 & 60.2 \\
\hline $\mathrm{CH}_{2} \mathrm{Cl}_{2}$ & 5.85 & 45.7 & Tetrahydrofuran & 5.49 & 26.9 \\
\hline $\mathrm{DMSO}$ & 5.08 & 144 & $\mathrm{t}$-Butylalcohol. & 5.77 & 17.8 \\
\hline $\mathrm{CH}_{3} \mathrm{COCH}_{3}$ & 4.95 & 50.1 & $1,4-\mathrm{C}_{4} \mathrm{H}_{8} \mathrm{O}_{2}$ & 6.00 & 25.7 \\
\hline Dimethyl formamide & 5.27 & 70.8 & $1,2-\mathrm{C}_{4} \mathrm{H}_{10} \mathrm{O}_{2}$ & 6.01 & 12.6 \\
\hline $\mathrm{C}_{4} \mathrm{H}_{8} \mathrm{O}$ & 5.80 & 37.1 & Carbon Disulphiode & 5.66 & 6.92 \\
\hline Nitrobenzene & 5.55 & 53.7 & $\mathrm{CH}_{3} \mathrm{COOH}$ & 5.96 & 10.7 \\
\hline $\mathrm{C}_{6} \mathrm{H}_{6}$ & 5.91 & 19.9 & $\mathrm{C}_{2} \mathrm{H}_{5} \mathrm{COOC} \mathrm{COH}_{5}$ & 5.58 & 18.6 \\
\hline $\mathrm{C}_{6} \mathrm{H}_{12}$ & 5.65 & 1.86 & & & \\
\hline
\end{tabular}

\section{Solvent Effect}

Rate constants of the oxidation of TLA in 18 solvents (Because of non-availability of all the parameters related to solvents, Carbon disulphide is not taken into account for calculations) are considered for calculation in LESR equation of Kamlett and Taft ${ }^{15}$ as follows:

$$
\log k_{2}=A_{0}+p \cdot \pi^{*}+b \cdot \beta+a \alpha
$$

In the above equation, $\pi^{*}$ is represented by solvent polarity data, $\beta$ is represented by the basicity of hydrogen bond acceptor and $\alpha$ is denoted by hydrogen bond donor acidities. $\mathrm{A}_{0}$ is known to be intercept. It is to be noted that out of eighteen solvents, twelve solvents have $\alpha$ values as zeros. Results of correlations are analyzed by the above equation are summarised below in equations six to nine.

$$
\begin{aligned}
& \log k_{2}=-3.60+1.55( \pm 0.20) \pi^{*}+0.12( \pm 0.16) \beta+0.07( \pm 0.16) \alpha \\
& \mathrm{R}^{2}=0.8439 ; \quad \mathrm{sd}=0.18 ; \quad \mathrm{n}=18 ; \quad \psi=0.40 \\
& \log k_{2}=-3.61+1.58( \pm 0.18) \pi^{*}+0.10( \pm 0.15) \beta \\
& \mathrm{R}^{2}=0.8416 ; \quad \mathrm{sd}=0.18 ; \quad \mathrm{n}=18 ; \quad \psi=0.39 \\
& \log k_{2}=-3.59+1.60( \pm 0.18) \pi^{*} \\
& \mathrm{r}^{2}=0.8371 ; \quad \mathrm{sd}=0.17 ; \quad \mathrm{n}=18 ; \quad \psi=0.41 \\
& \log k_{2}=-3.69+0.38( \pm 0.35) \beta \\
& \mathrm{r}^{2}=0.0703 ; \quad \mathrm{sd}=0.42 ; \quad \mathrm{n}=18 ; \quad \psi=0.99
\end{aligned}
$$

$\mathrm{n}$ is represented by the number of data points undertaken for study and $\psi$ is denoted to be the Exner mathematical parameter ${ }^{16}$.

Kamlet ${ }^{15}$ tri-parametric equation explains about $84 \%$ of data for solvent effect, which is not considered to the satisfactory measure as per the Exner ${ }^{16}$ Significant input is provided by $\pi^{*}$, which It on itself account about $82 \%$ of the data. $\beta \& \alpha$, are not contributing the significant roles.

Because of non-satisfactory results of the Kamlet model, the data is now put for analysis by Swain ${ }^{17}$ model of cation and anion solvation conception of solvent.

$$
\log k_{2}=\mathrm{aA}+\mathrm{bB}+\mathrm{C}
$$

$\mathrm{A}$ is representing anion solvating powers, $\mathrm{B}$ represents cation solvating powers and the notion $\mathrm{C}$ intercept data. The polarity of the solvents are represented by $(A+B)$. Rates of various solvent are subjected to analysis with the help of above equation (10), and with separate expressions of A \& B and with (A.+B.) also.

$\log k_{2}=0.67+( \pm 0.05) \mathrm{A}+1.63( \pm 0.03) \mathrm{B}-3.82$

$\mathrm{R}^{2}=0.9931 ; \mathrm{sd}=0.04 ; \mathrm{n}=19 ; \quad \psi=0.18$

$\log k_{2}=0.43( \pm 0.54) \mathrm{A}-3.70$

$\mathrm{r}^{2} \quad=0.0369 ; \mathrm{sd}=0.43 ; \mathrm{n}=19 ; \psi=1.00$ 
$\log k_{2}=1.58( \pm 0.12) \mathrm{B}-3.60$

$\mathrm{r}^{2}=0.9073 ; \mathrm{sd}=0.13 ; \mathrm{n}=19 ; \psi=0.31$

$\log k_{2}=1.31 \pm 0.13(\mathrm{~A}+\mathrm{B})-3.79$

$\mathrm{r}^{2} \quad=0.8637 ; \mathrm{sd}=0.16 ; \mathrm{n}=19 ; \psi=0.38$

The oxidation rate of TLA in various solvents expressed exceptional correlations with Swain model, in which the main contribution is of cation solvating powers. It is observed that it alone explains about $91 \%$ of results. Whereas anion solvating powers are not playing any role in the correlation analyses. $(\mathrm{A}+\mathrm{B})$ represented solvent polarity explains about $86 \%$ of results. Because of the high range of data explanation of data by solvent polarity, it is subjected to correlate with relative primitivity of solvent. A plot of rate with inverse of the relative permittivity is non-linear $\left(r^{2}=0.4838 ; \mathrm{sd}=0.32 ; \psi=0.74\right)$.

\section{Mechanism}

No effect of vinyl chloride indicated that the one-electron oxidation process by free radical mechanism is not operative in this reaction. The presence of a transition state is supported by Swain effect of solvent. The solvent effect also supported that the transition state is more polar than the reactant. Fractional order of less than one reaction order suggests that a thioester is formed as an intermediate in the reaction. Therefore, we propose that in the present reaction, thioester is formed as an intermediate which is decomposed in the rate-determining step.

The lack of any effect of radical scavengers, such as acrylonitrile on the reaction rate and the failure to induce the polymerization of acrylonitrile, point against the operation of a one-electron oxidation givingrise to free radicals. The observed solvent effect supports a transition state, which is more polar than the reactant state. The observed Michaelis-Menten type of kinetics with respect to thioacids also leads us to suggest the formation of thioester as an intermediate. It is, therefore, proposed that the reaction involves the formation of an ester intermediate and its subsequent decomposition in the slow step (Scheme-1). Cation solvating powers are also supporting the presence of a sulphenium cation in the slow step.

If one compares the reaction process of oxidation of TA by other halochromates like quinolinium fluorochromate $(\mathrm{QFC})^{18}$, quinolinium bromochromate $(\mathrm{QBC})^{19}$, morpholinium chlorochromate $(\mathrm{MCC})^{20}$ and (TPSD) ${ }^{21}$ and with IDC. QFC, TPSD and IDC representing a fractional order kinetic behaviour w.r.t. $\mathrm{TA}$, while the oxidation by $\mathrm{QBC}^{19}$ and $\mathrm{MCC}^{20}$ showed second-order reaction, unit with all reactants. It is because of the negligible value of $\mathrm{K}$ of thioester. All other parameters, such as the effect of acidity, are almost similar in all these reactions.

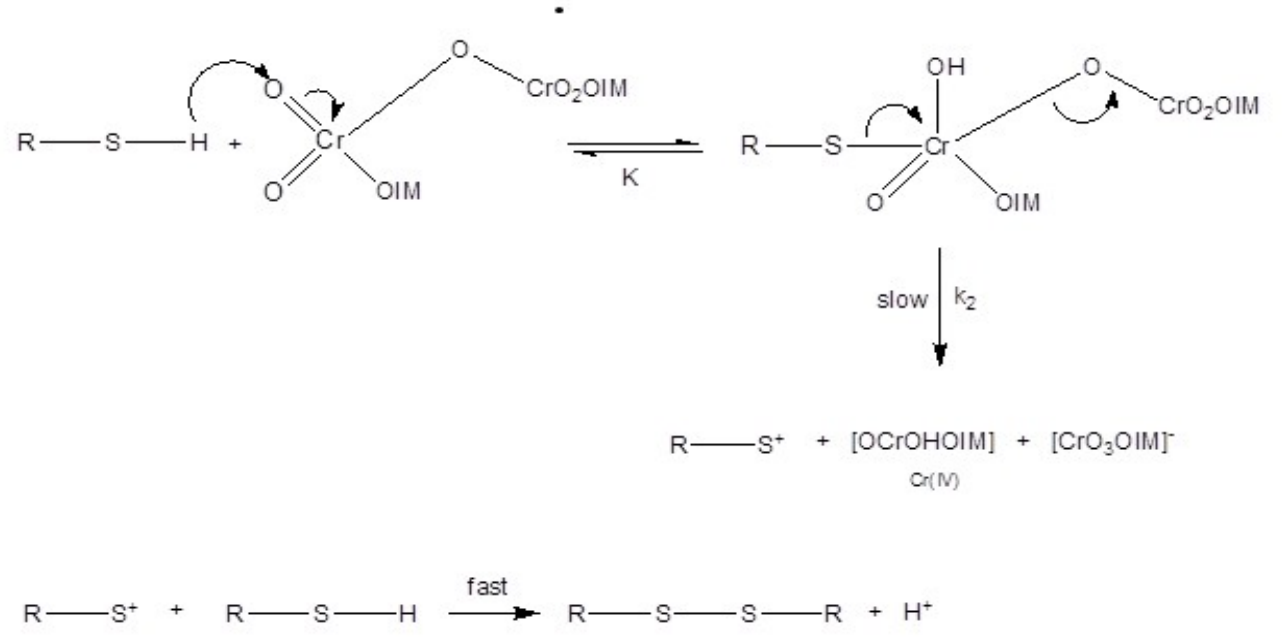

Scheme-1

CONCLUSION

It is concluded that the Involvement of a thioester formation in the slow step of the oxidation process of TA is also supported by the cation solvation powers of the solvent. 
RASĀYAN J. Chem.

Vol. 14 | No. 4 |2436-2441| October- December | 2021

\section{ACKNOWLEDGEMENT}

Dr. Anurag and Devendra Kumar are thankful to UGC, New Delhi, for monetary help as UGC-BSR Start-up grant and JRF respectively.

\section{REFERENCES}

1. E.J. Corey and W.J. Suggs, Tetrahedron Letters, 16(31), 2647(1975), https://doi.org/10.1016/S0040-4039(00)75204-X

2. F.S. Guziec and F.A. Luzzio, Synthesis, 9, 691(1980), https://doi.org/10.1055/s-1980-29172

3. M.N. Bhattacharjee, M.K. Choudhuri, H.S. Dasgupta, N. Roy, N. and D.T. Khathing, Synthesis, 7, 588(1982), https://doi.org/10.1055/s-1982-29872

4. A. Pandurangan, V. Murugesan and P. Palamichamy, Journal of Indian Chemical Society, 72, 479(1995).

5. S. Kim and DC Lhim, Bulletin of Chemical Society of Japan, 59, 3297(1986), https://doi.org/10.1246/bcsj.59.3297

6. M. Gilla, AK Meena, P. Swami, M. Baghmar and Vinita Sharma, Journal of Indian Chemical Society, 88(5), 699(2011).

7. N. Vyas, A. Daiya, A. Choudhary, M. Sharma and Vinita Sharma, European Chemical Bulletin, 2(11), 859(2013).

8. N. Vyas, G. Goswami, A. Choudhary, PTSRK Prasadrao and Vinita Sharma, International Journal of Chemistry, 4(3), 215(2015).

9. Itishree Hedau, K. Kanwar, S. Panwar, L. Mathur and Vinita. Sharma, Research Journal of Chemistry,1(1), 164(2018).

10. D.L. Leussing and I.M. Kolthoff, Journal of Electrochemical Society, 100, 334(1953), https://doi.org/10.1023/A:1005672505310

11. H. Krammer H., Journal of Association of Agriculture Chemistry, 35, 385(1952).

12. D.D. Perrin, W.L. Armarego and D.R. Perrin, Purification of Laboratory Chemicals, Pergamon Press, Oxford, (1966).

13. R.C. Kapoor, O.P. Kachhwaha and B.P. Sinha, Journal of Physical Chemistry, 73, 1627 (1969).

14. R.C. Kapoor, O.P. Kachhwaha and B.P. Sinha, Indian Journal of Chemistry, 10, 499 (1971).

15. M.J. Kamlet, J.L.M. Abboud, M.H. Abraham and R.W. Taft, Journal of Organic Chemistry, 48(17), 2877(1983), https://doi.org/10.1021/jo00165a018

16. O. Exner, Collection of Czechoslovak Chemical Communications, 31(8), 3222(1966).

17. C.G. Swain, S.H. Unger, N.R. Rosenquest and M.S. Swain, Journal of American Chemical Society, 105(3), 492(1983), https://doi.org/10.1021/ja00341a032

18. M. Khurana, P.K. Sharma and K.K. Banerji, Indian Journal of Chemistry, 37A(11), 1011(1998).

19. S. Vyas and P.K. Sharma, International Journal of Chemical Sciences, 2(1), 13(2004).

20. N. Malani, S. Pohani, M. Baghmar and P.K. Sharma, Indian Journal of Chemistry, 47A (9), 1373(2008).

21. Pradeep K. Sharma, International Journal of Chemistry, 2(3), 294(2013).

[RJC-6549/2021] 